\title{
The cholinergic anti-inflammatory pathway meets microRNA
}

\author{
Cell Research (2013) 23:1249-1250. doi:10.1038/cr.2013.128; published online10 September 2013
}

\begin{abstract}
Acetylcholine is a cellular mediator selected early in evolution as a key neurotransmitter, but its other biological roles including immune-modulation remain to be elucidated. In a recent paper published in Cell Research, Sun et al. report that acetylcholine modulates cytokine production in macrophages via microRNA-124.
\end{abstract}

The anti-inflammatory potential of the parasympathetic system has only been recently demonstrated $[1,2]$. The vagus nerve, the principal parasympathetic nerve connecting the nervous system with peripheral organs, can control inflammation in multiple settings of critical care [2]. Despite its recent discovery, multiple investigators have already reported that the vagus nerve can restrain systemic inflammation in experimental ischemia and reperfusion, hemorrhage, resuscitation, pancreatitis, colitis, endotoxemia, septic shock and severe sepsis. The vagus nerve controls systemic inflammation by inhibiting cytokine production in the splenic macrophages through a mechanism mediated by modulatory lymphocytes [3-5]. These studies indicate that acetylcholine, the principal neurotransmitter of the vagus nerve, is also produced by the immune system and serves as a bidirectional molecular signal for the neural-immune coordination.

Acetylcholine inhibits cytokine production in macrophages via $\alpha 7$ nicotinic acetylcholine receptor ( $\alpha 7 \mathrm{nAChR})$, and nicotine is a more stable agonist to control cytokine production $[6,7]$. Studies with actinomycin $\mathrm{D}$ and cycloheximide indicated that the anti-inflammatory potential of acetylcholine is independent of de novo protein synthesis, and is mediated by the cholinergic inhibition of NF- $\kappa B$ [6]. Likewise, nicotine inhibits the NF- $\kappa$ B pathway via STAT3 but independently of the traditional STAT3 transcriptional modulation [8, 9]. Despite controversy on the STAT3 phosphorylation, all the studies indicate that STAT3 protein expression is critical for the cholinergic inhibition of the NF-kB pathway [8-10]. The role of STAT3 in the cholinergic anti-inflammatory pathway was first shown in postoperative paralytic ileus, a common postsurgical pathology characterized by the inflammation of the intestinal muscularis. Intestinal manipulation causes paralytic ileus, gastroparesis and intestinal inflammation characterized by phosphorylated STAT3-positive cells in the serosal site of the bowel wall, most probably granulocytes and monocytes recruited by the tissue trauma. Significantly, vagus nerve stimulation prevents paralytic ileus in control mice but not in the LysM-Stat $3^{\mathrm{H} /}$ - mice lacking STAT3 protein in their myeloid cell lineage including macrophages [8].

Recent studies with $\alpha 7 \mathrm{nAChR}$ knockout mice indicate that nicotine can signal through multiple receptors, but choline is a specific $\alpha 7 \mathrm{nAChR}$ agonist [9]. Nicotine and choline inhibit LPSinduced STAT3 Tyr705 phosphorylation, similar to that reported for JAK2 inhibitor or stattic, a typical inhibitor of STAT3 tyrosine phosphorylation $[9,10]$. These findings suggest that the cholinergic anti-inflammatory pathway is independent of the STAT3 tyrosine phosphorylation, but it requires STAT3 protein expression, suggesting that unphosphorylated STAT3 (U-STAT3) mediates the anti-inflammatory po- tential of $\alpha 7 \mathrm{nAChR}$. In line with these findings, U-STAT3 can bind to NF- $\kappa$ B in

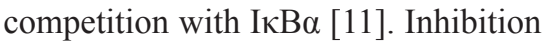
of LPS-induced STAT3 tyrosine phosphorylation by $\alpha 7 \mathrm{nAChRs}$ increases the proportion of U-STAT3 available to inhibit NF- $\kappa \mathrm{B}$ and its inflammatory responses. However, unlike the inhibition of STAT3 tyrosine phosphorylation, STAT3 protein depletion prevents the physiological implications of U-STAT3 and the cholinergic anti-inflammatory pathway of $\alpha 7 \mathrm{nAChR}$. These results challenge the current perspective of USTAT3 as an inactive protein, and suggest that inhibition of STAT3 tyrosine phosphorylation does not necessarily indicate an inactivation of all STAT3 functions, but rather, a modulation of its pathway. Indeed, the binding of USTAT3 to NF- $\mathrm{kB}$ may prevent activation of TNF transcription by NF- $\kappa \mathrm{B}$, but the resulting STAT3-NF- $\kappa$ B complex may enhance the production of other factors [11].

In a recent paper in Cell Research, Sun et al. [10] show that nicotine can inhibit both STAT3 tyrosine phosphorylation and protein expression by inducing the microRNA-124 (miR-124) via $\alpha 7$ nAChR. miR-124 agomir inhibits STAT3 protein levels and LPS-induced IL6 and TNF production. Interestingly, miR-124 appears to inhibit TNF release by preventing the translation of TNFconverting enzyme (TACE), a protease required for the processing of the membrane-anchored precursor pro-TNF (Figure 1). This study provides promising results showing that pretreatment with miR-124 agomir improves the 24 $\mathrm{h}$ survival rate in murine endotoxemia. Given that sepsis is characterized by a 


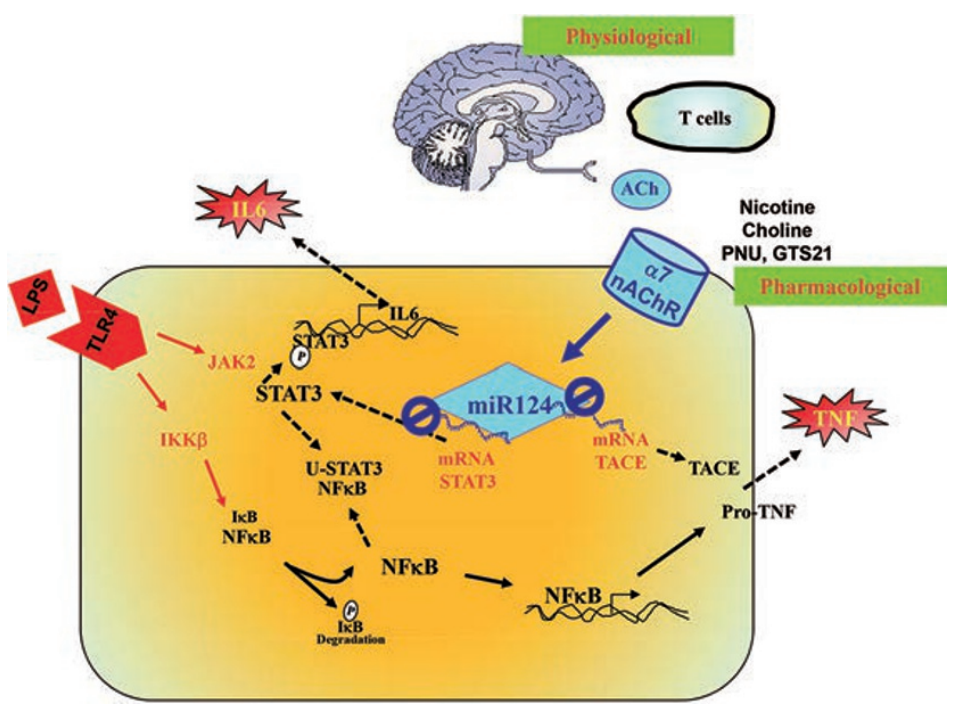

Figure $1 \mathrm{miR}-124$ plays a critical role in the cholinergic anti-inflammatory pathway of $\alpha 7 n A C h R$. Bacterial entodotoxin activates both IKK $\beta-N F-\kappa B$ and JAK2-STAT3 pathways, triggering the production of TNF and IL6. Physiological activation of the vagus nerve or modulatory lymphocytes releases acetylcholine to modulate the production of these inflammatory factors. Previous studies indicated that $\alpha 7 \mathrm{nAChR}$ can inhibit the NF-kB pathway by preventing LPS-induced STAT3 tyrosine phosphorylation. Unphosphorylated U-STAT3 can bind and inhibit p65 NF-KB. Cholinergic physiological or pharmacological activation of $\alpha 7 \mathrm{nAChR}$ induces the production of miR-124, which inhibits the translation of the STAT3 mRNA and subsequent IL6 production. Additionally, miR-124 also inhibits the translation of the TACE mRNA and subsequent TNF production.

late mortality of many days after the infection, future studies with longer survival times will be needed to determine whether miR-124 agomir merely delays the pathogenesis of endotoxemia or induces a lasting protection. In addition to endotoxemia, this treatment will need to be tested on other experimental models that are more clinically relevant such as cecal ligation and puncture. Unlike endotoxemia, the pathogenesis of cecal ligation and puncture is characterized by both polymicrobial infection and necrotic tissue.

Micro noncoding RNAs (miRNAs) have recently emerged as important regulators of gene expression in a wide variety of biological processes. Similar modulatory mechanism has just been proposed for long noncoding RNAs (lncRNAs) [12]. With 5 000-10 000 miRNAs expressed in mammalian cells, they can modulate over $60 \%$ of the protein-coding genes. miRNAs are noncoding transcripts of $18-25$ nucleotides processed in the nucleus by the RNase Drosha. Large pre-miRNAs translocate to the cytoplasm where the miRNA hairpin is cleaved by the endoribonuclease Dicer. The resulting single RNA strands bind to the $3^{\prime}$-untranslated regions (UTRs) of specific mRNA to inhibit their protein transcription. This complex and energyconsuming mechanism appears to be justified and designed to provide two significant advantages: it quickly stops mRNA translation and "tunes" gene expression of a specific set of genes. Faster and greater ability to stop mRNA translation and degradation is a critical process to quickly decrease the acute expression of potentially dangerous inflammatory cytokines such as TNF and IL6. The expression of these factors needs to be strong to trigger proper immune responses, but it has to be quickly reduced to minimize collateral tissue damage. miRNAs provide an efficient mechanism to accelerate the inhibition of these factors. However, miRNAs do not represent an efficient mechanism to completely switch gene expression off, but rather, it usually functions as a "tuning" mechanism modulating gene expression in a range of 1-4 folds. This is a typical characteristic of the cholinergic anti-inflammatory pathway, which takes advantage of the chemical instability and short life of acetylcholine and miRNAs to quickly modulate inflammation and to avoid tissue damage but keeps a baseline expression against infection. These mechanisms can provide biological advantages versus the immunosuppressive effects of glucocorticoids. miRNAs can allow the cholinergic anti-inflammatory pathway to temper the acute production of inflammatory factors and limit potential overzealous secondary responses while keeping a controlled inflammatory surveillance.

\section{Luis Ulloa ${ }^{1}$}

${ }^{1}$ Center of Immunology and Inflammation, Department of Surgery, New Jersey Medical School, Rutgers University, NJ 07103, USA Correspondence: Luis Ulloa

E-mail: Mail@LuisUlloa.com

\section{References}

1 Ulloa L. Nat Rev Drug Discov 2005; 4:673684.

2 Tracey KJ. Nat Immunol 2010; 11:561-564.

3 Huston JM, Ochani M, Rosas-Ballina M, et al. J Exp Med 2006; 203:1623-1628.

4 Peña G, Cai B, Ramos L, et al. J Immunol 2011; 187:718-725.

5 Rosas-Ballina M, Olofsson PS, Ochani M, et al. Science 2011; 334:98-101.

6 Wang H, Liao H, Ochani M, et al. Nat Med 2004; 10:1216-1221.

7 Wang $\mathrm{H}, \mathrm{Yu} \mathrm{M}$, Ochani $\mathrm{M}$, et al. Nature 2003; 421:384-388.

8 de Jonge WJ, van der Zanden EP, The FO, et al. Nat Immunol 2005; 6:844-851.

9 Peña G, Cai B, Liu J, et al. Eur J Immunol 2010; 40:2580-2589.

10 Sun Y, Li Q, Gui H, et al. Cell Res 2013; 23:1270-1283.

11 Yang J, Liao X, Agarwal MK, et al. Genes Dev 2007; 21:1396-1408.

12 Carpenter S, Aiello D, Atianand MK, et al. Science 2013; 341:789-792. 